\title{
Community Acquired Pneumonia Among Pediatric Outpatients in Salvador, Northeast Brazil, with Emphasis on the Role of Pneumococcus
}

Cristiana M.C. Nascimento-Carvalho, Antônio A. Lopes, Maria Daniela B.S. Gomes, Morgana P. Magalhães, Juliana R. Oliveira, Ana Luisa Vilas-Boas, Roberta Ferracuti, Maria Cristina C. Brandileone, Maria Luiza L.S. Guerra, Noraney N. Alves, Ledilce A. Athayde, Renilza M. Caldas, Maria Goreth M. A. Barberino, Jussara Duarte, Maria Angélica S. Brandão, Heonir Rocha, Yehuda Benguigui, and José Luis Di Fabio

\author{
Departments of Pediatrics and of Internal \\ Medicine, Clinical Epidemiology Unit, \\ Faculty of Medicine, Professor Hosannah de \\ Oliveira Pediatric Center, Federal University \\ of Bahia; Aliança Hospital; Central Laboratory \\ of Bahia, Salvador, Bahia; Bacteriology \\ Division, Adolfo Lutz Institute, São Paulo, \\ Brazil; and Pan American Health Organization \\ Washington DC, USA.
}

\begin{abstract}
Pneumonia is one of the leading causes of hospitalization and death among children in developing countries, and mortality due to pneumonia has been associated with $S$. pneumoniae infection. This investigation was designed to describe the antimicrobial susceptibility and serotype patterns of pneumococcal strains recovered from the blood of children with community-acquired pneumonia (CAP) and to assess the clinical findings of pneumococcal bacteremic patients with pneumonia. In a 26 month prospective study, blood cultures were obtained as often as possible from children $(<16$ years of age) diagnosed with CAP in two emergency rooms. Antimicrobial drug susceptibility tests and serotyping were performed when pneumococcus was identified. We studied 3,431 cases and cultured blood samples from $65.5 \%$ of those. Pneumococcus was recovered from $0.8 \%$ of the blood samples. The differences in age, somnolence, wheezing and hospitalization among children with and without pneumococcal bacteremia were statistically significant. Pneumococcal bacteremia was age-related (mean 1.63 \pm 1.55 ; median 0.92 ) and associated with somnolence and hospitalization among children with CAP. One strain was recovered from pleural fluid. Penicillin resistance was detected in $21.0 \%(4 / 19)$ of the strains at an intermediate level, whereas $63.0 \%$ of the strains were resistant to trimethoprim-sulfamethoxazole. The most common serotypes were 14 and $6 \mathrm{~B}$, and these serotypes included the resistant strains. Eight of our 18 isolates from blood were of types included in the heptavalent conjugate pneumococcal vaccine, recently licensed in the USA.

Key Words: Streptococcus pneumoniae, antimicrobial resistance, serotypes, blood culture, community-acquired pneumonia, children.
\end{abstract}

Received on 22 September 2000; revised 5 December 2000. Address for correspondence: Dr. Cristiana NascimentoCarvalho, Department of Pediatrics, Faculty of Medicine, Federal University of Bahia, Rua Prof. Aristides Novis No.105/ 1201B - Salvador, Bahia, Brazil. Zip Code: 40210-730. E-mail: otaviocr@svn.com.br. This study was supported by the Pan American Health Organization.

The Brazilian Journal of Infectious Diseases 2001;5(1):13-20 (C) 2001 by The Brazilian Journal of Infectious Diseases and Contexto Publishing. All rights reserved.

$1413-8670$
Acute respiratory infection (ARI), particularly pneumonia, continues to be one of the leading causes of hospitalization and death among children in most developing countries [1-3]. Since mortality from preventable diseases has diminished markedly as the result of worldwide systematic application of oral rehydration therapy and vaccination, pneumonia is one of the challenges to face at the present time $[4,5]$. According to several studies, mortality due to ARI is associated with bacterial infections, caused especially 
by $S$. pneumoniae and $H$. influenzae [6-9]. Since the early reports from Boston in 1965, Australia in 1967, and South Africa in 1977, penicillin-nonsusceptible pneumococci have been reported with increasing frequency of infection around the world [10-16], including Latin America [17-21]. It has become increasingly important, therefore, to consider the use of the conjugate pneumococcal vaccine which is immunogenic in children over 2 months of age [22]. The efficacy of an adequate pneumococcal vaccine will depend on the existence of detailed, reliable and representative data on prevalence and serotype distribution of $S$. pneumoniae in the region where the vaccine is to be used [23]. Since 1993, the Pan American Health Organization has been conducting an Epidemiological Surveillance Study to determine the relative prevalence of capsular types of $S$. pneumoniae which cause invasive disease, particularly pneumonia, and their patterns of antimicrobial resistance in Latin America (SIREVA-VIGIA) [23]. In Brazil, reports of the bacteriologic characteristics of $S$. pneumoniae are limited.[18,24,25] In Salvador, a large city in Northeast Brazil, there is no information on the antimicrobial susceptibility and distribution of serotypes of pneumococcal strains isolated from children diagnosed with pneumonia. The purposes of the present investigation are to provide such information and to assess the clinical findings of pneumococcal bacteremic patients with pneumonia.

\section{Materials and Methods}

\section{$\underline{\text { Study design and population }}$}

We conducted a prospective study as part of the SIREVA-VIGIA in Salvador, in Northeast Brazil [23], from September, 1997, to October, 1999. We attempted to enroll all identified cases of pneumonia among children seen in the Emergency Room (ER) of the Professor Hosannah de Oliveira Pediatric Center (PHOPC) and the Pediatric ER of the Aliança Hospital (AH) in Salvador. Data collection was based on a questionnaire that asked for demographic and clinical findings. A large proportion $(64.3 \%)$ of the questionnaires were filled out immediately after each case identification by the primary physician. The diagnosis of pneumonia was based on either the WHO case definition criteria (ie., cough and tachypnea) [26], and/or on crackles on auscultation and/or on radiological findings. Chest $\mathrm{x}$-ray was read by the duty pediatrician during the consultation. All collected data and all emergency room records were reviewed by the research group members once a week beginning in March, 1998, at the PHOPC, and in May, 1998, at the AH. Retrospectively, the same group also completed the questionnaires for those patients characterized as having pneumonia whose questionnaires were not completed prospectively. Each case was crossreferenced with the Bacteriology Laboratory log of the respective hospital, in order to verify the collection of blood and/or pleural fluid for culture.

\section{$\underline{\text { Bacteriologic data }}$}

One blood culture and/or pleural fluid culture was obtained in as many cases as possible, before the start of the antimicrobial therapy. Data concerning the use of immediately previous antibiotic therapy was collected, but only intravenous antibiotic treatment was used as a criterion for exclusion.

At the PHOPC laboratory, the blood and pleural fluid specimens $(1.0 \mathrm{ml}$ to $3.0 \mathrm{ml}$ of blood or pleural fluid) were immediately inoculated into $30 \mathrm{ml}$ of Brain Heart Infusion broth with SPS $(0.025 \%)$ and incubated at $35^{\circ} \mathrm{C}$. All broths were sub-cultured after $24 \mathrm{hrs}, 48$ hrs and at 7 days of incubation onto Brain Heart Infusion agar with 5\% sheep blood and on chocolate agar at $35^{\circ} \mathrm{C}$. At the $\mathrm{AH}$ laboratory, $0.5 \mathrm{ml}$ to $4.0 \mathrm{ml}$ of the collected specimens were immediately inoculated into $20 \mathrm{ml}$ of supplemented Brain Heart Infusion broth and incubated in Bact/Alert Organon at $35^{\circ} \mathrm{C}$ for 7 days. Whenever the Bact/Alert Organon displayed a positive result, the broth was sub-cultured onto Columbia agar with $5 \%$ sheep blood and onto chocolate agar at $35^{\circ} \mathrm{C}$ in a $5 \% \mathrm{CO}_{2}$ incubator for 18 hrs to $24 \mathrm{hrs}$. Streptococcus pneumoniae was distinguished from other $\alpha$-haemolytic streptococci by 
using the bile solubility test and the optochin disc test. The research group members were informed about all isolated bacteria from children attending both ERs in order to cross-reference them with the clinical records. The strains isolated during the study were sent to the Central Laboratory of Bahia (LACEN) and then by airplane to the Adolfo Lutz Institute, in São Paulo, where the bacteriologic identification was rechecked and the antimicrobial drug susceptibility tests and serotyping were performed. Pneumococcal resistance to penicillin was screened initially by using a $1 \mu \mathrm{g}$ oxacillin disc. Penicillin resistance was considered probable with an oxacillin zone size less than $20 \mathrm{~mm}$. Disc-diffusion was used for surveillance of resistance to other antibiotics including chloramphenicol, trimethoprim-sulfamethoxazole, erythromycin, clindamycin, ofloxacin, vancomycin, and tetracycline. The non-susceptible strains by oxacillin disc were submitted for the determination of minimal inhibitory concentration (MIC) testing against penicillin by using the broth microdilution method (with MuellerHinton broth supplemented with $2 \%$ to $5 \%$ lysed horse blood) (NCCLS, 2000) [27]. Seroptyping of pneumococcus was done by the Neufeld-Quellung reaction using sera produced by the Statens Seruminstitut, Copenhagen, Denmark.

\section{Study setting}

The PHOPC is a public university hospital located in a central area of Salvador, the capital of Bahia, in Northeast Brazil. The population of Salvador is approximately 2,800,000 inhabitants. The PHOPC serves children of predominantly lower socioeconomic status who live in Salvador and adjacent cities. The AH is a general private hospital located in a middle class area of Salvador. Its Pediatric ER attends children from middle to middle-upper and high socio-economic status. The physicians in both ERs were all pediatricians trained to use the WHO case definition of pneumonia, and they were all asked to collect blood and/or pleural fluid for culture whenever a patient was eligible for this study after receiving the consent of the child's guardian.

\section{Data analysis}

Statistical analyses were performed by using the Statistical Package for the Social Sciences (SPSS 9.0). Differences in proportions were assessed by the Pearson Chi Square test or Fisher's exact test, as appropriate. Means of continuous variables were compared by Mann-Whitney U. Confidence Interval (95\%) was reported for mean difference. The statistical tests were two tailed, with a significance level of 0.05 . The study was approved by the institutional review board of each hospital and by the Ethics Committee of the Faculty of Medicine of the Federal University of Bahia.

\section{Results}

By active surveillance, a total of 3,431 cases of pneumonia among 3,221 children were identified during the study period. These totals were comprised of 2,476 cases from the PHOPC and 955 cases from the $\mathrm{AH}$. The intervals between the separate episodes among the patients with more than 1 infection were, in all instances, greater than 1 month. One hundred seventy of the patients had 2 or more separate episodes of pneumonia. Of all patients, $54.3 \%$ were male and $45.7 \%$ were female. The median age was 1.92 years (range 2 days to 15.5 years, mean $2.87 \pm 2.78$ years). Tachypnea and crackles were reported in $57.7 \%$ and $68.3 \%$ of the cases, respectively. Chest $\mathrm{x}$-ray was performed in $93.2 \%$, and radiologically confirmed infiltrate was reported in $96.0 \%$. Considering the radiological pattern, the infiltrates were classified as interstitial $(24.7 \%)$, alveolar $(51.0 \%)$ or mixed (interstitialalveolar) (20.3\%). All children received antimicrobial therapy. Blood cultures were done in $73.7 \%$ (704/ $955)$ at the $\mathrm{AH}$, and in $62.3 \%(1,542 / 2,476)$ at the PHOPC (overall 65.5\%). Contamination was detected in $4.1 \%$ of the blood culture sets. The frequency of isolating $S$. pneumoniae from blood was $0.8 \%(18 / 2,246)[0.9 \%(14 / 1,542)$ at the PHOPC, $0.6 \%(4 / 704)$ at the AH; $=0.4]$. The 
Table 1. Significant differences among children with community acquired pneumonia when blood culture was performed

\begin{tabular}{|c|c|c|c|c|}
\hline \multirow[b]{2}{*}{ Characteristic } & \multicolumn{2}{|c|}{ Pneumococcus } & \multirow[b]{2}{*}{ Total } & \multirow[b]{2}{*}{ p value } \\
\hline & Yes & No & & \\
\hline \multicolumn{5}{|l|}{ Age (years) } \\
\hline Mean \pm SD & $1.63 \pm 1.55$ & $2.91 \pm 2.79$ & $2.90 \pm 2.78$ & 0.044 \\
\hline Median & 0.92 & 2.00 & 2.00 & \\
\hline range & $0.17-5.92$ & $0.02-15.50$ & $0.02-15.50$ & \\
\hline Wheezing $\dagger$ & $11.8(2 / 17)$ & $47.5(935 / 1967)$ & $47.2(937 / 1984)$ & 0.003 \\
\hline Somnolence $\dagger$ & $50.0(8 / 16)$ & $19.1(357 / 1870)$ & $19.4(365 / 1886)$ & 0.005 \\
\hline Hospitalization $\dagger$ & $66.7(12 / 18)$ & $29.3(653 / 2228)$ & $29.7(666 / 2246)$ & 0.0005 \\
\hline
\end{tabular}

$\dagger$ Results are reported in $\%(\mathrm{n} / \mathrm{N})$.

Table 2. Frequency of serotypes and antimicrobial resistance to penicillin of $S$. pneumoniae isolates recovered in sterile fluids from children with pneumonia

\begin{tabular}{lcccc}
\hline & \multicolumn{2}{c}{ Hospital } & & \\
\cline { 2 - 3 } Serotype & PCPHO & AH & Total (\%) \\
\hline 1 & 1 & 0 & 1 & $(5.3)$ \\
5 & 1 & 0 & 1 & $(5.3)$ \\
$6 \mathrm{~A}$ & 1 & 1 & 2 & $(10.5)$ \\
$6 \mathrm{~B}$ & 1 & $2\left(1^{*}\right)$ & 3 & $(15.7)$ \\
$9 \mathrm{~N}$ & 1 & 0 & 1 & $(5.3)$ \\
$10 \mathrm{~A}$ & 1 & 0 & 1 & $(5.3)$ \\
14 & $4\left(3^{*}\right)(1 \dagger)$ & 0 & 4 & $(21.0)$ \\
$15 \mathrm{II}$ & 1 & 1 & 2 & $(10.5)$ \\
$19 \mathrm{~A}$ & 2 & 0 & 2 & $(10.5)$ \\
20 & 1 & 0 & 1 & $(5.3)$ \\
$23 \mathrm{~F}$ & 1 & 0 & 1 & $(5.3)$ \\
\hline Total & $\mathbf{1 5}$ & $\mathbf{4}$ & $\mathbf{1 9}(\mathbf{1 0 0 . 0})$ \\
\hline
\end{tabular}

*Penicillin non-susceptible strains.

$\uparrow$ Strain isolated from pleural fluid, penicillin susceptible.

ISSerotypes in serogroup 15 were not distinguished. 
significant differences in demographic and clinical findings among the groups of children with and without pneumococcus as identified from blood cultures are shown in Table 1 . The mean difference of age between those groups was 1.28 (95\% CI 0.5 - 2.06). No significant differences were noticed in the frequency of tachypnea, crackles, cough, ability to drink, chest retraction, fever (axillary temp $>37.5{ }^{\circ} \mathrm{C}$ during the consultation), chronic underlying illness, day care center attendance, chest $\mathrm{x}$-ray performance, radiological infiltrate patterns, or gender. Antimicrobial use during the 72 hours before the blood was obtained for culture was reported in $17.6 \%$ of the cases and such information was not reported on $22.8 \%$ of the questionnaires. Streptococcus pneumoniae was isolated from 1 patient who reported the use of trimethoprimsulfamethoxazole $(1 / 305,0.3 \%)$. The isolation rate among patients who reported no use of antimicrobials during 72 hours before the collection of blood for culture was $0.9 \%(13 / 1,428)(p=0.49)$. We also observed that $1.8 \%(12 / 666)$ of the hospitalized patients had pneumococcus isolated from blood culture in comparison to $0.4 \%(6 / 1,580)$ of the patients who were not hospitalized $(\mathrm{p}=0.001)$.

Nineteen strains of pneumococcus were isolated during the study period; 18 were isolated from blood and 1 was isolated from pleural fluid (blood culture was not drawn from this child). Penicillinresistance was detected in $21.0 \%$ (4/19) of the strains isolated from different children living in different quarters of Salvador, all of them at the intermediate level; 3 of the resistant strains were isolated from children visiting the PHOPC, and 1 was isolated from a child visiting the AH. The 3 resistant strains isolated at the PHOPC were serotype 14 , and the 1 resistant strain isolated at the AH was serotype 6B (Table 2). The most prevalent serotype was 14 , the same serotype most frequently resistant in this study. All isolates were sensitive to chloramphenicol, clindamycin, ofloxacin, tetracycline, erythromycin and vancomycin, but only $37.0 \%$ of the strains were sensitive to trimethoprim-sulfamethoxazole.

\section{Discussion}

We found a $0.8 \%$ yield of pneumococcus in blood cultures. This rate was similar to those reported in several studies of the etiology of pediatric community acquired pneumonia(CAP). [28-32] The overwhelming majority of cases of CAP during childhood are caused by respiratory viruses [33]. Streptococcus pneumoniae has been estimated, by serological methods, to be responsible for one third of all cases of acute pneumonias $[9,31,32]$. However, the proportion of children with pneumonia who are bacteremic at the time blood is obtained for culture is uncertain [34]. Some data are available from studies of concurrent cultures of blood and lung aspirate. Blood cultures were positive in only $13.0 \%$ of patients with bacterial pneumonia, documented by lung punctures, as reported by Silverman, et al. [35]. Although the bacteriologic procedures were not the same at both laboratories, the difference of isolates was not statistically significant.

The present study provided evidence that bacteremia among children with CAP was age-related (Table 1). This fact may be related to the immaturity of childrens' immune systems during the first years of life. Somnolence and hospitalization were associated with pneumococcal bacteremia, whereas wheezing was not. This finding is reasonable since hospitalized patients usually appear more severely ill than do those who are managed as outpatients, and somnolence is regarded as a severity sign. It was expected, therefore, that hospitalized and somnolent patients would have a greater chance of being bacteremic. On the contrary, wheezing was associated with nonbacteremic pneumococcal cases. Perhaps this can be explained by the association of wheezing with infection by respiratory viruses [36].

We found that $17.6 \%$ of the patients were using antimicrobial drugs before the collection of blood. It is widely known that the previous use of antimicrobial drugs can decrease the isolation rate of pathogenic bacteria. The difference between the proportions of pneumococci isolated as related to previous antimicrobial use was not statistically significant, probably because of the small number of strains studied. 
The use of trimethoprim-sulfamethoxazole did not interfere with the isolation of $S$. pneumoniae from 1 child; this strain was susceptible to that drug but compliance could not be certified.

Until now, the results of pneumococcal susceptibility tests and serotyping in this study are in accord with data from other Brazilian states (Table 2) $[18,24,25]$. These results suggest that penicillin G can still be used as initial therapy to treat children in our region diagnosed with pneumococcal pneumonia. Penicillin remains adequate to treat pneumonia caused by $S$. pneumoniae with an intermediate level of resistance to penicillin [37]. The 19 strains studied were distributed among 11 serotypes; the most frequent serotypes were $14,6 \mathrm{~B}$, $6 \mathrm{~A}, 19 \mathrm{~A}$ and serogroup 15 . The penicillinnonsusceptible strains $(14,6 \mathrm{~B})$ were among the most frequent serotypes. These data agree with results reported by Ko, et al. [38], who studyed 221 pneumococcal isolates recovered in cerebrospinal fluid from patients with meningitis in Salvador, when infections with serotypes 14 and $6 \mathrm{~B}$ were responsible for $83.0 \%$ of the 29 penicillin non-susceptible cases (all intermediate level). By comparing the serotype distribution of our pneumococcal strains with those represented in the heptavalent pneumococcal conjugate vaccine recently licensed in the USA [39], we found that 3 of our 11 serotypes $(6 \mathrm{~B}, 14,23 \mathrm{~F})$ were contained in that vaccine, 2 of the $5(14,6 \mathrm{~B})$ most frequent, the same penicillin-nonsusceptible ones. Although there are 90 capsular types of pneumococcus, relatively few types are responsible for most disease in children [33], with types 6A, 6B, 14 and 19A commonly found in pediatric disease worldwide. The predominance of penicillin-resistant serotypes $6 \mathrm{~B}$ and 14 have been described worldwide [17-21, 40-42].

It is important to emphasize that caution must be taken in interpreting the features of $S$. pneumoniae reported here because of the small number of strains studied. Culturing blood was not feasible for children seen in the ER of the PHOPC before this investigation, when blood was drawn only from hospitalized patients. Blood culturing was achieved due to the support provided for this study.
The increasing prevalence of penicillinnonsusceptible $S$. pneumoniae has influenced the decision to order blood cultures for patients with pneumonia [43]. In spite of the low rate of isolation in this study $(0.8 \%)$, blood culture remains the gold standard for the diagnosis of pneumococcal pneumonia $[43,44]$; it is also the most practical way to identify the causative organism, mainly when data derived from susceptibility testing and serotype patterns are the goals.

\section{Acknowledgements}

We thank Robert Austrian, M.D., for reviewing this manuscript, Rogério Santos-Jesus, M.D., for his technical assistance in the analysis, and every pediatrician, nurse, laboratory technician and medical student working in the Emergency Rooms of the participating hospitals for collaborating on this study.

\section{References}

1. Denny F.W., Loda F.A. Acute respiratory infections are the leading cause of death in children in developing countries. Am J Trop Med Hyg 1986;35:1-2.

2. Benguigui Y. Infecções Respiratórias Agudas: Fundamentos Técnicos das Estratégias de Controle. Washington, D.C.: OPS, c1997, p2.

3. Berman S., McIntosh K. Selective primary health care: strategies for control of disease in the developing world. XXI Acute Respiratory Infections. Rev Infect Dis 1985;7:674.

4. PAHO/WHO. ARI in the Americas - Biannual Reports $\mathrm{N}^{\circ}$ 1, December 1995. PAHO/HCP/HCT/ARI/95.27, 1995.

5. PAHO/WHO. Notícias sobre IRA, nº 29, 1996.

6. Burman L.A., Norrby R., Trollfors B. Invasive pneumococcal infections: incidence, predisposing factors, and prognosis. Rev Infect Dis 1985; 7:133-42.

7. Shann F. Etiology of severe pneumonia in children in developing countries. Pediatr Infect Dis J 1986;5:247-52.

8. Turner R.B., Lande A.E., Chase P., et al. Pneumonia in pediatric outpatients: cause and clinical manifestations. J Pediatr 1987;111(2):194-200.

9. Heiskanen-Kosma T., Korppi M., Jokinen C., et al. Etiology of childhood pneumonia: serologic results of a prospective, population-based study. Pediatr Infect Dis J 1998; 17(11):986-91. 
10. Hansman D., Bullen M.M. A resistant pneumococcus. Lancet 1967;2:264-5.

11. Jacobs M.R., Koornhof H.J., Robins-Brown R.M., et al. Emergence of multiple resistant pneumococci. N Engl J Med 1978;229:735-40.

12. Appelbaum P.C. Antimicrobial resistance in Streptococcus pneumoniae: An overview. Clin Infect Dis 1992; $15: 77-83$.

13. Hofmann J., Cetron M.S., Farley M.M., et al. The prevalence of drug-resistant Streptococcus pneumoniae in Atlanta. NEngl J Med 1995;333:481-6.

14. Pallares R., Linares J., Vadillo M., et al. Resistance to penicillin and cephalosporin and mortality from severe pneumococcal pneumonia in Barcelona, Spain. N Engl J Med 1995;333:474-80.

15. Butler J.C., Hofmann J., Cetron M.S., et al. The continued emergence of drug-resistant Streptococcus pneumoniae in the United States: an update from the Centers for Disease Control and Prevention's Pneumococcal Sentinel Surveillance System. J Infect Dis 1996; 174:986-93.

16. CDC. Defining the public health impact of drug-resistant Streptococcus pneumoniae: Report of a working group. MMWR 1996; $45: 1-20$.

17. Rossi A., Ruvinsky R., Regueira M., et al. Distribution of capsular types and penicillin-resistance of strains of Streptococcus pneumoniae causing systemic infections in Argentinian children under 5 years of age. Microbial Drug Resist 1997;3:135-40.

18. De Cunto Brandileone M.C., Diaz Vieira V.S., Casagrande S.T., et al. Prevalence of serotypes and antimicrobial resistance of Streptococcus pneumoniae strains isolated from Brazilian children with invasive infections. Microbial Drug Resist 1997;3:141-6.

19. Castañeda E., Leal A.L., Castillo O., et al. Distribution of capsular types and antimicrobial susceptibility of invasive isolates of Streptococcus pneumoniae in Colombian children. Microbial Drug Resist 1997;3:147-52.

20. Echániz-Aviles G., Velázquez-Meza M.E., Carnalla-Barajas M.N., et al. Antimicrobial susceptibilities and capsular types of invasive Streptococcus pneumoniae isolated in children in Mexico City. Microbial Drug Resist 1997;3:153-7.

21. Hortal M., the Pneumococcus Study Group. Capsular type distribution and susceptibility to antibiotics of Streptococcus pneumoniae clinical strains isolated from Uruguayan children with systemic infections. Microbial Drug Resist 1997;3:159-63.

22. Eskola J., Anttila M. Pneumococcal conjugate vaccines. Pediatr Infect Dis J 1999; 18:543-51.

23. Di Fabio J.L., Homma A., Quadros C. Pan American Health Organization Epidemiological Surveillance Network for Streptococcus pneumoniae. Microbial Drug Resist 1997;3(2):131-3.
24. Levin A.S., Teixeira L.M., Sesselogo J.F., Barone A.A. Resistance of Streptococcus pneumoniae to antimicrobials in São Paulo, Brazil: clinical features and serotypes. Rev Inst Med Trop S Paulo 1996;38: 187-92.

25. Sessegolo J.F., Levin A.S., Levy C.E., et al. Distribution of serotypes and antimicrobial resistance of Streptococcus pneumoniae strains isolated in Brazil from 1988 to 1992. J Clin Microbiol 1994;32(4):906-11.

26. World Health Organization. Case management of acute respiratory infections in developing countries: report of a working group meeting. Document WHO/RSD/ 85.15. Rev 1. Geneva: WHO, 1985.

27. National Committee for Clinical Laboratory Standards. Methods for dilution antimicrobial susceptibility tests for bacteria that grow aerobically. Villanova, PA $7^{\text {th }}$ ed. M7-A2.: NCCLS, 2000.

28. Ramsey B.W., Marcuse E.K., Foy H.M., et al. Use of bacterial antigen detection in the diagnosis of pediatric lower respiratory tract infections. Pediatrics 1986;78:1-9.

29. Claesson B.A., Trollfors B., Brolin I., et al. Etiology of community-acquired pneumonia in children based on antibody responses to bacterial and viral antigens. Pediatr Infect Dis J 1989;8:856-62.

30. Nohynek H., Eskola J., Laine E., et al. The causes of hospital-treated lower respiratory tract infection in children. Am J Dis Child 1991;145:618-22.

31. Juven T., Mertsola J., Waris M., et al. Etiology of community-acquired pneumonia in 254 hospitalized children. Pediatr Infect Dis J 2000;19:293-8.

32. Wubbel L., Muniz L., Ahmed A., et al. Etiology and treatment of community-acquired pneumonia in ambulatory children. Pediatr Infect Dis J 1999; 18:98-104.

33. Klein J.O. Bacterial Pneumonias. In: Feigin R.D., Cherry J.D. Textbook of Pediatric Infectious Diseases. WB Saunders Co, Philadelphia, 1998:273-84.

34. Baraff L.J. Blood culture in children with pneumonia. Ann Emerg Med 1996;27:774-6.

35. Silverman M., Stratton D., Diallo A., et al. Diagnosis of acute bacterial pneumonia in Nigerian children: value of needle aspiration of lung and countercurrent immunoelectrophoresis. Arch Dis Child 1977;52:925-31.

36. Tupasi T.E., Lucero M.G., Magdangal D.M., et al. Etiology of acute lower respiratory tract infection in children from Alabang, Metro Manila. Rev Infect Dis 1990;12(S8):S929-39.

37. Friedland I.R., McCracken G.H., Jr. Management of infections caused by antibiotic-resistant Streptococcus pneumoniae. N Engl J Med 1994;331:377-82.

38.Ko A.I., Reis J.N., Coppola S.J., et al. Clonally-related penicillin non-susceptible Streptococcus pneumoniae serotype 14 from cases of meningitis in Salvador, Brazil. Clinical Infect Dis 2000;30:78-86. 
39. Black S., Shinefield H., Fireman B., et al. Efficacy, safety and immunogenicity heptavalent pneumococcal conjugate vaccine in children. Pediatr Infect Dis J 2000;19:187-95.

40. Broome C.V., Facklam R.R., Allen J.R., et al. Epidemiology of pneumococcal serotypes in the United States. J Infect Dis 1980; 141:119-23.

41. Eskola J., Takala A.K., Kela E., et al. Epidemiology of invasive pneumococcal infections in children in Finland. JAMA 1992;268:3323-7.

42. Scott J.A.G., Hall A.J., Dagan R., et al. Serogroup-specific epidemiology of Streptococcus pneumoniae: Associations with age, sex, and geography in 7,000 episodes of invasive disease. Clin Infect Dis 1996;22:973-81.

43. Bryan C.S. Blood Cultures for Community-acquired Pneumonia. No Place to Skimp! Chest 1999;116(5):1153-4.

44. Austrian R., Gold J. Pneumococcal bacteremia with especial reference to bacteremic pneumococcal pneumonia. Ann Intern Med 1964;60:759-76. 